\title{
Propriedades tecnológicas da madeira de eucalipto submetida ao tratamento térmico
}

\author{
Cláudio Gumane Francisco JUIZO ${ }^{1 *}$, Linéia Roberta ZEN ${ }^{1}$, Walderson KLITZKE ${ }^{1}$, \\ Morgana Cristina FRANÇA ${ }^{1}$, Victor Gonçalves CREMONEZ ${ }^{1}$, Ricardo Jorge KLITZKE ${ }^{2}$ \\ ${ }^{1}$ Programa de Pós-Graduação em Engenharia Florestal-DETF, Universidade Federal do Paraná, Curitiba, Brasil. \\ ${ }^{2}$ Professor Associado-DETF, Universidade Federal do Paraná, Curitiba, Brasil. \\ *E-mail: c.gumane@gmail.com
}

Recebido em setembro/2017; Aceito em maio/2018.

RESUMO: Este trabalho teve o objetivo de avaliar as propriedades tecnológicas da madeira de Eucalyptus sp tratada termicamente. Foram utilizadas amostras de madeira previamente secas até $12 \%$ de umidade, as quais foram separadas em cinco tratamentos, sendo um testemunha e quatro condições de exposição sob elevadas temperaturas e tempo, utilizando um forno cerâmico. As amostras foram separadas para avaliação das propriedades químicas, físicas e mecânicas. Nos resultados obtidos, observou-se decréscimo do teor de extrativos totais e holoceluloses, enquanto o teor de lignina de Klason aumentou com a temperatura e tempo de exposição. Da mesma forma, os tratamentos térmicos causaram acréscimo da perda de massa e da efetiva repelência de água, com redução da massa específica aparente, taxa de absorção de água e higroscopicidade da madeira. $\mathrm{Na}$ resistência das peças verificou-se aumento do módulo de elasticidade (MOE) e diminuição do modulo de ruptura (MOR) com aplicação de tratamentos térmicos. Na compressão paralela verificou-se aumento do MOE e MOR nas peças tratadas, servindo de parâmetros para possibilidades de uso estrutural da madeira até ao estado limite último. A tensão de Dureza Janka decresceu com aplicação dos tratamentos térmicos limitando a utilização dos mesmos em projetos que requerem maior resistência na superfície.

Palavras-chave: Temperatura, Propriedades químicas, Propriedades físicas, Propriedades mecânicas.

\section{Technological properties of the eucalyptus wood under the heat treatment}

\begin{abstract}
This work aimed to evaluate the technological properties of the heat treated wood of Eucalyptus sp. Were used wood samples Pre-dried up to $12 \%$ of moisture, which were separated into five treatments, one control and four diferente exposure conditions under high temperatures and time using a ceramic kiln. The samples were separated for evaluation of chemical, physical and mechanical properties. In the obtained results there was a decrease in total extractive and holocelluloses contents, while the Klason lignin content increased with the temperature and exposure times. In the same way, the heat treatments caused an increase of the mass loss and the effective water repellency with reduction of the apparent specific gravity, rate of water absorption and hygroscopicity of the wood. In the resistance of the pieces, the modulus of elasticity (MOE) was increased and the modulus of rupture (MOR) was reduced under the heat treatments. In the Parallel compression was verified increasing of MOE and MOR of the heat treated samples, serving as parameters for possibilities of structural use of the wood up to the last limit condition. The Janka hardness tensile decreased with the application of heat treatments, limiting the use of the wood in projects that require higher surface resistance.
\end{abstract}

Keywords: Temperature, Chemical properties, Physical properties, Mechanical properties.

\section{INTRODUÇÃO}

As madeiras de Eucalipto têm sido largamente utilizadas no mercado, em resultado da sua versatilidade como matériaprima para obtenção de diversos produtos. No entanto, apesar da crescente demanda por essas espécies, a grande variabilidade entre as mesmas, ainda é um fator limitante para a utilização em alguns setores, como fabricação de embalagens, construção civil e movelaria para uso em ambiente externo, no qual a madeira está sujeita a constante variações ambientais.

Pois a variabilidade ambiental entre espécies, observadas nas madeiras de eucalipto, fazem com que haja necessidade de algumas melhorias tecnológicas, que vão além do melhoramento genético e práticas silviculturais, para alterar as características estruturais e tecnológicas em função da aplicação que se pretende da madeira em serviço.

Dentre várias melhorias que são feitas, Brito et al. (2008) destacam tratamento utilizando calor, conhecidos como modificação térmica, termorretificação ou retificação térmica, como sendo um dos mais importantes tratamentos atualmente utilizados, podendo ser controlado para não causar alterações significativas nas propriedades estruturais da madeira.

De acordo com Sundqvist (2004); Brito et al. (2008); Kocaefe et al. (2008) neste tratamento, a madeira é submetida a altas temperaturas $\left(100\right.$ a $\left.280^{\circ} \mathrm{C}\right)$ em função do tempo de exposição, causando modificações na composição química, devido a decomposição das moléculas de celuloses, hemiceluloses e lignina, reduzindo a acessibilidade dos grupos hidroxilas responsáveis pela higroscopicidade da madeira, e consequentemente aumento da perda de massa pela redução do material lenhoso.

Boonstra et al. (2007); Winandy; Rowell (2005), reportam que o aumento da temperatura, causa redução acentuada das hemiceluloses com correspondente perda da resistência. Já Winandy; Rowell (2005), afirmam que durante a degradação térmica da madeira, observa-se perda de massa, que resulta na 
perda de resistência mecânica, com diminuição de xilose, galactose e a arabinose.

Além da redução da acessibilidade dos grupos hidroxilas devido a decomposição das moléculas de hemiceluloses, neste processo ocorre também a redução das propriedades de resistência da madeira. Porém, segundo Kocaefe et al. (2008), essa situação só acontece em níveis de temperatura considerados mais elevados (acima de $200^{\circ} \mathrm{C}$ ) pois abaixo dessa temperatura, ocorre o entrecruzamento da lignina e a cristalinidade da celulose, podendo manter estável a resistência mecânica da madeira.

Segundo Silva (2012); Garcia et al. (2012), a madeira tratada termicamente tem sido utilizada em vários continentes, sendo largamente comercializada, na Europa, Estados Unidos, Canadá, onde são empregados processos já patenteados, como Retification ${ }^{\circledR}$ (França), Bois Perdure ${ }^{\circledR}$ (França e Canadá), Oil Heat Treatment-OHT ${ }^{\circledR}$ (Alemanha), Plato ${ }^{\circledR}$ Wood (Países Baixos), ThermoWood ${ }^{\circledR}$ (Finlândia, Suíça e Canadá) e Westwood (Estados Unidos, Canadá e Rússia).

No Brasil, alguns processos já patenteados estão sendo introduzidos para fazer face a sustentabilidade ambiental, visando a utilização da madeira de eucalipto, sem aplicação de produtos preservantes na melhoria durabilidade natural.

Nas técnicas de tratamento térmico já introduzidas e atualmente usadas no mercado, além do calor e do controle da umidade inicial da madeira, empregam-se outras etapas, como a utilização de oxigênio, nitrogênio, vapor e óleos, encarecendo o processo. Porém a possibilidade de utilização de técnicas de que reduzem custos, sem causar grandes alterações nas propriedades pode ser uma alternativa viável para indústria.

Assim sendo, foi desenvolvido este trabalho para avaliar o efeito da modificação térmica nas propriedades tecnológicas da madeira de Eucalyptus sp, utilizando um forno em sistema fechado, com total ausência de gás, vapor e óleos, como uma alternativa para o setor madeireiro.

\section{MATERIAL E MÉTODOS}

Para realização do estudo foram utilizadas tábuas de Eucalyptus sp, com dimensões de 650x110x25mm (comprimento, largura e espessura) as quais foram previamente secas em estufa convencional até $12 \%$ de umidade.

Após a secagem e acondicionamento, as tábuas foram aplainadas e reduzidas em peças com dimensões nominais de $350 \times 60 \times 22 \mathrm{~mm}$ (comprimento, largura e espessura) que foram utilizadas para os tratamentos térmicos utilizando parâmetros de temperatura e tempo de exposição descritos por Cademartori et al. (2015) como se observa na Tabela 1.

Tabela 1. Tratamentos térmicos utilizados para exposição da madeira de Eucalyptus sp.

Table 1. Thermal treatments used to exposure the wood of Eucalyptus sp.

\begin{tabular}{cc}
\hline Tratamento & Temperatura $\left({ }^{\circ} \mathrm{C}\right) /$ Tempo de exposição (horas) \\
\hline $\mathrm{T}_{1}$ & Testemunha \\
$\mathrm{T}_{2}$ & $180 / 4$ \\
$\mathrm{~T}_{3}$ & $210 / 2$ \\
$\mathrm{~T}_{4}$ & $210 / 4$ \\
$\mathrm{~T}_{5}$ & $240 / 2$ \\
\hline
\end{tabular}

Assim sendo, os tratamentos térmicos das amostras foram realizados em forno cerâmico da marca Linn Elektro Therm, modelo $k k 260$ com temperatura máxima de até $1340^{\circ} \mathrm{C}$. E para cada tratamento foram alocadas 40 peças totalizando 200 amostras incluindo o tratamento testemunha.

Após os tratamentos térmicos, as amostras foram acondicionadas em câmara climática para estabilização da umidade e avaliação das propriedades químicas, físicas e mecânicas.

\subsection{Propriedades químicas}

Para avaliação das propriedades químicas as amostras foram trituradas em um moinho de faca, para promover maior contato da madeira com os solventes químicos, em seguida o material triturado foi classificado em uma peneira de $60 \mathrm{mesh}$, para realização das análises.

Em seguida determinou-se o teor de extrativos totais, teor de lignina de Klason e teor de holoceluloses, de acordo com procedimentos recomendados pela American Society for Testing and Materials - (ASTM, 2007).

\subsection{Propriedades físicas}

As propriedades físicas foram avaliadas por meio da determinação da perda de massa, massa especifica aparente, taxa de absorção de água e efetiva repelência de água.

A perda de massa da madeira foi avaliada em amostras acondicionadas, utilizando valores de pesagens obtidos antes e depois dos tratamentos térmicos, segundo a metodologia proposta por Cademartori et al. (2012).

Seguiu-se a avaliação da massa específica aparente utilizando procedimentos da Associação Brasileira de Normas Técnicas - ABNT (1997), em corpos de prova obtidos de cada tratamento com dimensões de 50X25X25mm (comprimento, largura e espessura).

A taxa de absorção de água, determinou-se com auxílio da Equação 1, após a imersão de cada tratamento, em água a temperatura de $25 \pm 2^{\circ} \mathrm{C}$ durante 24 horas.

$$
\mathrm{TAA}=\frac{\mathrm{Mu}-\mathrm{M} 12 \%}{\mathrm{M} 12 \%} \times 100 \quad \text { (Equação 1) }
$$

em que: T: Taxa de absorção de água das amostras; Mu: Massa das amostras após imersão em água; M12\%: Massa das amostras médidas a $12 \%$ de umidade.

Com base nos valores de taxa de absorção de água determinada das amostras termorretificada e testemunhas, calculou-se a efetividade de repelência à água dos tratamentos térmicos, com o auxílio da equação 2 .

$$
\mathrm{ERA}=\frac{\mathrm{T} 1-\mathrm{T} 2}{\mathrm{~T} 1} \times 100
$$

(Equação 2)

em que: ERA: Efetividade de repelência à água das amostras; T1: Taxa de absorção de água das amostras não termorretificadas; T2: Taxa de absorção de água das amostras termorretificadas.

\subsection{Propriedades mecânicas}

Para determinação das propriedades mecânicas foram analisados o módulo de elasticidade (MOE) e módulo de ruptura (MOR) em flexão estática, e compressão paralela seguindo a recomendação da norma ASTM (2000), assim como a tensão máxima em Dureza Janka fazendo adaptação da norma (ABNT 1997). Os corpos de prova foram ensaiados utilizando uma máquina universal de ensaios da EMIC com capacidade para 10Tf equipada com uma célula de carga com capacidade de $500 \mathrm{kgf}$. 


\subsection{Análise de dados}

Os dados obtidos foram tabulados no Excel e analisados em Statgraphics centurion $X V$, no qual fez-se o teste de homogeneidade e análise de variância dos tratamentos térmicos, e quando detectada diferença significativa entre os mesmos, foi empregado o teste de Tukey a 5\% de significância.

Para complementar, foram feitas análises de variação da perda de resistência em flexão estática e compressão paralela, em função da perda de massa observada na madeira de cada tratamento de modificação térmica.

\section{RESULTADOS}

\subsection{Propriedades químicas}

Observa-se na Tabela 2, que os tratamentos térmicos ocasionaram alteração na composição química da madeira, e as análises estatísticas indicaram diferenças significativas no teor de lignina de Klason e teor de holoceluloses, com exceção do teor de extrativos totais que foi estatisticamente igual entre os tratamentos testados.

Tabela 2. Valores médios dos componentes químicos avaliadas em cada tratamento térmico da madeira de Eucalyptus sp.

Table 2. Mean values of the chemical components evaluated in the heat treatments of Eucalyptus sp.

\begin{tabular}{cccccc}
\hline Componentes & \multicolumn{5}{c}{ Tratamento } \\
\cline { 2 - 6 } químicos & $\mathrm{T}_{1}$ & $\mathrm{~T}_{2}$ & $\mathrm{~T}_{3}$ & $\mathrm{~T}_{4}$ & $\mathrm{~T}_{5}$ \\
\hline TLK (\%) & $30,24 \mathrm{c}$ & $47,09 \mathrm{~b}$ & $46,55 \mathrm{~b}$ & $49,89 \mathrm{~b}$ & $58,48 \mathrm{a}$ \\
TH (\%) & $64,45 \mathrm{a}$ & $48,25 \mathrm{~b}$ & $46,31 \mathrm{~b}$ & $43,38 \mathrm{~b}$ & $35,81 \mathrm{c}$ \\
TET (\%) & $5,31 \mathrm{a}$ & $4,66 \mathrm{a}$ & $7,13 \mathrm{a}$ & $6,73 \mathrm{a}$ & $5,72 \mathrm{a}$ \\
\hline
\end{tabular}

TLK: Teor de lignina de Klason; TH: Teor de Holoceluloses; TET: Teor de extrativos totais; Médias seguidas pela mesma letra minúscula na linha não diferem entre si pelo teste de Tukey.

\subsection{Propriedades físicas}

$\mathrm{Na}$ Tabela 3, os tratamentos térmicos ocasionaram decréscimo significativo da massa especifica aparente e taxa absorção de água, sendo que a perda de massa e a efetividade de repelência à água aumentaram de forma significativa, com o acréscimo das temperaturas e tempo de exposição da madeira de Eucalyptus sp.

Tabela 3. Valores médios das Propriedades físicas avaliadas em cada tratamento térmico da madeira de Eucalyptus sp.

Table 3. Mean values of the physical properties evaluated in the heat treatments of Eucalyptus sp.

\begin{tabular}{lccccc}
\hline Prop. & \multicolumn{5}{c}{ Tratamento } \\
\cline { 2 - 6 } & $\mathrm{T}_{1}$ & $\mathrm{~T}_{2}$ & $\mathrm{~T}_{3}$ & $\mathrm{~T}_{4}$ & $\mathrm{~T}_{5}$ \\
\hline MEA $\left(\mathrm{g} / \mathrm{cm}^{3}\right)$ & $0,73 \mathrm{a}$ & $0,68 \mathrm{a}$ & $0,64 \mathrm{ab}$ & $0,62 \mathrm{~b}$ & $0,61 \mathrm{~b}$ \\
PM $(\%)$ & - & $9,15 \mathrm{~b}$ & $15,02 \mathrm{ab}$ & $19,76 \mathrm{ab}$ & $23,9 \mathrm{a}$ \\
TAA $(\%)$ & $11,4 \mathrm{a}$ & $10,53 \mathrm{a}$ & $4,16 \mathrm{~b}$ & $4,33 \mathrm{~b}$ & $5,05 \mathrm{~b}$ \\
ERA $(\%)$ & - & $19,27 \mathrm{~b}$ & $63,57 \mathrm{a}$ & $62,30 \mathrm{a}$ & $55,7 \mathrm{a}$ \\
\hline
\end{tabular}

MEA: Massa específica aparente; PM: Perda de massa; TAA: Taxa de absorção de água; ERA: Efetividade de repelência à água; Médias seguidas pela mesma letra minúscula na linha não diferem entre si pelo teste de Tukey.

\subsection{Propriedades mecânicas}

Para as propriedades mecânicas apresentados na Tabela 4 , pode-se observar que os tratamentos térmicos causaram aumento do módulo de elasticidade, porém sem diferença estatística entre os tratamentos. Já, para o módulo de ruptura houve um decréscimo dos valores, com diferença estatística entre os tratamentos em flexão estática e compressão paralela, assim como, na tensão máxima em Dureza Janka avaliado na superfície da madeira.
A variação das propriedades de resistência em flexão estática e compressão paralela as fibras da madeira, em função da perda de massa ocorrida termorretificação está apresentada na Figura 1. Pode-se observar na Figura 1, que a variação da perda de massa ocorrida com a termorretificação influenciou na redução da resistência mecânica da madeira de Eucalyptus $\mathrm{sp}$, e apesar de se ter observado melhoria na rigidez da madeira, a resistência das peças tratadas reduziu significativamente em função da perda de massa.

Tabela 4. Valores médios das Propriedades mecânicas de cada tratamento térmico da madeira de Eucalyptus sp.

Table 4. Mean values of the mechanical properties of each heat treatment of Eucalyptus sp.

\begin{tabular}{cccccc}
\hline Prop. & \multicolumn{5}{c}{ Tratamento } \\
\cline { 2 - 6 } & $\mathrm{T}_{1}$ & $\mathrm{~T}_{2}$ & $\mathrm{~T}_{3}$ & $\mathrm{~T}_{4}$ & $\mathrm{~T}_{5}$ \\
\hline$f e \mathrm{MOE}$ & $11857 \mathrm{a}$ & $12435 \mathrm{a}$ & $12914 \mathrm{a}$ & $11118 \mathrm{a}$ & $12449 \mathrm{a}$ \\
$(\mathrm{MPa})$ & $*(0,18)$ & $*(0,12)$ & $*(0,09)$ & $*(0,26)$ & $*(0,18)$ \\
$f e \mathrm{MOR}$ & $90 \mathrm{a}$ & $70 \mathrm{~b}$ & $67 \mathrm{~b}$ & $57 \mathrm{~b}$ & $24 \mathrm{c}$ \\
$(\mathrm{Mpa})$ & $*(0,22)$ & $*(0,23)$ & $*(0,25)$ & $*(0,40)$ & $*(0,30)$ \\
$f c \mathrm{MOE}$ & $31223 \mathrm{c}$ & $48116 \mathrm{ab}$ & $42373 \mathrm{abc}$ & $51709 \mathrm{a}$ & $35830 \mathrm{bc}$ \\
$(\mathrm{Mpa})$ & $*(0,44)$ & $*(0,12)$ & $*(0,39)$ & $*(0,18)$ & $*(0,38)$ \\
$f c \mathrm{MOR}$ & $498 \mathrm{~b}$ & $602 \mathrm{a}$ & $530 \mathrm{~b}$ & $502 \mathrm{~b}$ & $498 \mathrm{~b}$ \\
$(\mathrm{Mpa})$ & $*(0,058)$ & $*(0,033)$ & $*(0,17)$ & $*(0,12)$ & $*(0,09)$ \\
$D J \sigma$ & $545 \mathrm{a}$ & $376 \mathrm{~b}$ & $333 \mathrm{~b}$ & $315 \mathrm{~b}$ & $307 \mathrm{~b}$ \\
$(\mathrm{Mpa})$ & $*(016)$ & $*(0,14)$ & $*(0,20)$ & $*(0,17)$ & $*(0,19)$ \\
\hline
\end{tabular}

$f e$-flexaõ estática (Mpa); $f c$ : compressão paralela (Mpa); DJ: Dureza janka; $\sigma$ : tensão máxima $(\mathrm{Mpa}) .{ }^{*}$ coeficiente de variação; Médias seguidas pela mesma letra minúscula na linha não diferem entre si pelo teste de Tukey.

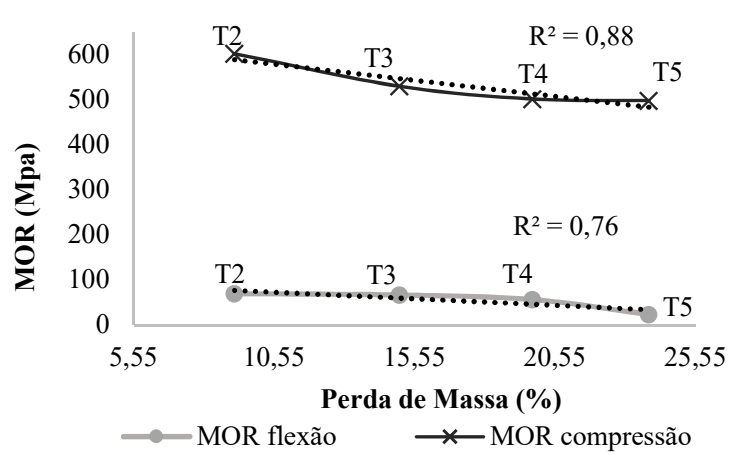

Figura 1. Variação das propriedades de resistência em função da perda de massa de cada tratamento térmico.

Figure 1. Variation of the strength properties as a function of the mass loss in each heat treatment

\section{DISCUSSÃO}

4.1Propriedades químicas

Pode-se observar na Tabela 2, que as madeiras submetidas aos tratamentos térmicos apresentaram maiores teores de lignina em relação ao tratamento testemunha. Sendo que o tratamento a $240^{\circ} \mathrm{C}$ foi o que se destacou, com um aumento de $93,39 \%$ no teor de lignina de Klason, em relação ao tratamento testemunha.

Os tratamentos térmicos não ocasionam a formação de lignina na madeira. No entanto, a elevação da temperatura causa a degradação de moléculas de hemiceluloses e celuloses, que se volatizam, tornando maior a acessibilidade e porcentual da lignina no balanço do material. Situação semelhante foi observada por Brito et al. (2008); Poubel et al. (2013); Batista et al. (2016), segundo os quais o aumento dessa substância, é observada pela degradação de outros componentes químicos 
principalmente as hemiceluloses quando a madeira é submetida à elevadas temperaturas.

Os teores de lignina de Klason observados neste estudo são semelhantes aos valores reportados por Silva (2012), que determinou, $44 \%, 51,7 \%$ e $53,9 \%$ na madeira de $E$. citriodora, submetida a $200^{\circ} \mathrm{C}, 220^{\circ} \mathrm{C}$ e $240^{\circ} \mathrm{C}$ respectivamente.

$\mathrm{O}$ teor de holocelulose, apresentou decréscimo substancial com aumento do tempo e temperatura na realização dos tratamentos térmicos. E para Sundqvist (2004) a degradação das heloceluloses começa a ser significante a partir de $140^{\circ} \mathrm{C}$, sendo que acima dessa temperatura a degradação se intensifica. Razão pela qual, se verificou-se uma redução de aproximadamente $50 \%$ no teor de holoceluloses, no tratamento a $240^{\circ} \mathrm{C}$ por 2 horas.

Assim sendo, os valores médios observados são semelhantes aos obtidos por Moura et al. (2012) no lenho de compressão de E. grandis (46\%) e por Silva (2012) que verificou uma variação de $42,2 \%$ a $52,5 \%$ na madeira de $E$. citriodora.

Em relação ao teor de extrativos apresentados na Tabela 2, é possível notar uma redução no tratamento a $180^{\circ} \mathrm{C}$ e um aumento significativo nos tratamentos mais intensos, Situação semelhante as observações de Sundqvist (2004), Poubel et al. (2013); Zanuncio et al. (2014), segundo os quais a redução observada, é resultado da volatilização dos extrativos polares que aparecem em grande quantidade na madeira da maioria nas folhosas.

Ainda assim, o posterior aumento no teor de extrativos a temperaturas mais intensas que foi observado na Tabela 2, segundo Moura et al. (2012); Zanuncio et al. (2014), é devido a degradação de outros componentes, como os carboidratos (arabinose, galactose, gannose, xylose), que posteriormente reagem, se transformando em novos extrativos na madeira.

\subsection{Propriedades físicas}

A massa específica aparente (Tabela 3 ) teve uma tendência a diminuição com o aumento da temperatura, atingindo redução em torno de $16,43 \%$ em relação ao tratamento testemunha, na exposição à $240^{\circ} \mathrm{C}$ por duas horas.

Os tratamentos térmicos não causaram alteração da ultraestrutura da parede para redução da massa específica aparente, pois segundo Carrasco et al. (2016) a mesma só ocorre devido à degradação das hemiceluloses e elevada perda de água nas interfaces entre moléculas de celulose, resultando na redução do material lenhoso e consequente perda de massa da madeira.

Cademartori et al. (2015) utilizando a mesma faixa de temperatura reportou valores relativamente baixos de massa específica aparente, variando de $\left.0,38 \mathrm{~g} / \mathrm{cm}^{3}-0,41 \mathrm{~g} / \mathrm{cm}^{3}\right)$. Porém, o porcentual de perda de massa encontrado foi semelhante ao do presente estudo.

Por outro lado, Silva (2012) obteve valores relativamente superiores na massa especifica aparente de madeiras de $E$. citriodora tratadas com temperaturas variando de $160^{\circ} \mathrm{C}$ e $240^{\circ} \mathrm{C}$. Sendo que, Carrasco et al. (2016); Cademartori et al. (2012) obtiveram $0,607 \mathrm{~g} / \mathrm{cm}^{3}$ e $0,634 \mathrm{~g} / \mathrm{cm}^{3}$ na massa especifica aparente e $12,72 \%$ e $22,51 \%$ na perda de massa respectivamente, à semelhança do que foi observado no presente estudo. Contrariamente ao observado na massa específica aparente, a perda de massa apresentou uma relação direta com a temperatura, verificando-se aumento de até $23,90 \%$ nos tratamentos mais intensos.
A degradação moléculas de hemiceluloses, tornou o efeito irreversível dos tratamentos térmicos mais notável, se manifestando pela redução da taxa de absorção de água, principalmente na exposição à $210^{\circ} \mathrm{C}$ por duas horas, onde se observou a menor taxa de absorção possível.

A efetiva repelência de água, seguiu a mesma tendência observada para taxa de absorção de água, e os seus valores estão dentro dos limites estabelecidos por Modes (2010), segundo a qual os tratamentos térmicos contribuíram para o aumento na estabilidade dimensional da madeira.

Apesar do aumento da porosidade devido a degradação de substâncias constituintes do material lenhoso durante os tratamentos térmicos, Poubel et al. (2013); Moura et al. (2012); Cademartori et al. (2012), afirmam que a redução da higroscopicidade e consequentemente aumento da efetiva repelência a água, é resultado da quebra de ligações de hidrogênio que ocorre pela degradação das moléculas de hemiceluloses, e para Moura et al. (2012) pode ser uma vantagem na comercialização, uma vez que diminui o seu peso reduzindo os custos de transporte.

\subsection{Propriedades de mecânicas}

$\mathrm{O}$ ensaio de flexão estática, resultou no maior valor absoluto do MOE na exposição da madeira a temperaturas de $210^{\circ} \mathrm{C}$ por duas horas, sem diferenças significativas entre os tratamentos testados (Tabela 4). No entanto, para o MOR, foram observadas diferenças significativas, decrescendo de forma abrupta a medida que a temperaturas e tempo de exposição da madeira foi aumentando, chegando a se verificar $73,34 \%$ de redução no tratamento à $240^{\circ} \mathrm{C}$ por duas horas.

Segundo Araújo et al. (2012), o MOE e MOR são propriedades que se correlacionam com a massa específica da madeira, fato que não se observou nesse estudo, pois a redução da massa especifica não resultou na redução do MOE.

Os valores de MOE obtidos neste estudo, são relativamente maiores que os resultados observados por Araújo et al. (2012), testando diferentes métodos para tratamento térmico de madeira, os quais obtiveram valores máximos de 7560,8 Mpa utilizando prensa, 7495,9Mpa na estufa a vácuo e $10028 \mathrm{Mpa}$ na estufa a vácuo com nitrogênio.

Por outro lado, os valores do MOR foram relativamente semelhantes aos resultados de Araújo et al. (2012) que determinaram valores máximos de 77,10Mpa, 91,66Mpa e $114,15 \mathrm{Mpa}$ nos tratamentos térmicos na prensa, estufa a vácuo e estufa a vácuo com nitrogénio respectivamente.

Na compressão paralela, verificou-se situação semelhante a do teste de flexão estática no qual o MOE foi maior na exposição da madeira à $210^{\circ} \mathrm{C}$ por quatro horas enquanto que o MOR foi menor tratamento testemunha. Tanto no MOE assim como no MOR, verificou-se efeito significativo da temperatura na redução das propriedades de resistência e rigidez da madeira respetivamente.

E o aumento que se observa do MOE tanto em flexão estática assim como em compressão paralela, pode estar associado a cristalização das moléculas de celulose que ocorre até temperaturas de $200^{\circ} \mathrm{C}$ (Hakkou et al., 2005). E como era esperado o decréscimo do MOR devido a perda de massa ocorrida com aplicação de $210^{\circ} \mathrm{C}$ e $240^{\circ} \mathrm{C}$ no experimento.

A tensão em Dureza Janka foi afetada pelos tratamentos térmicos, verificando-se redução significativa da mesma com o aumento da temperatura e tempo de exposição, chegando a se observar perdas de $43,70 \%$ da resistência. 
A medida que se elevou temperatura $\left(180^{\circ} \mathrm{C}\right.$ e $210^{\circ} \mathrm{C}$ e $240^{\circ} \mathrm{C}$ ), a degradação de substâncias de material lenhoso foi mais intensa e progressiva, deixando a madeira desses tratamentos perdeu com menos massa e mais porosa na superfície. Segundo Yildiz et al. (2006), essa alteração torna a madeira mais frágil, resultando em elevadas perdas de resistência nas mais variadas propriedades.

Na relação entre as propriedades da madeira e a perda de massa, ilustrada na figura 1 para flexão estática, pode se observar que a elevação da temperatura até $240^{\circ} \mathrm{C}$ por 2 horas, causou redução da rigidez, com perdas que chegam à $74 \%$ em relação ao tratamento testemunha. E uma correlação positiva de $76 \%$ entre o MOR e perda de massa da madeira.

Ainda na figura 1, para a compressão paralela, a temperatura de $180^{\circ} \mathrm{C}$ resultou em um acréscimo ligeiro, e ligeira perda de massa, no entanto a utilização de temperaturas mais intensas, resultou num decréscimo da rigidez, com correlações de $88 \%$ entre o MOR e a perda de massa respetivamente.

\section{CONCLUSÕES}

Com base nos resultados obtidos na madeira termorretificada de Eucalyptus sp, conclui-se que:

$\mathrm{O}$ aumento da temperatura e tempo de exposição, aumentam o teor de lignina de Klason, na medida que reduzem o teor de holoceluloses e extrativos totais na madeira.

Ha redução da taxa de absorção de água e da efetiva repelência de água na madeira, conferindo a madeira melhor estabilidade dimensional.

Verificou-se aumento da perda de massa e redução da massa específica da madeira com a termorretificação da madeira.

A termorretificação, melhora as propriedades de resistência, na medida que diminuem as propriedades de rigidez e tensão em Dureza Janka da madeira.

\section{REFERÊNCIAS}

ARAÚJO, S. de O.; VITAL B. R.; MENDOZA, Z. M. dos S. H. de; VIEIRA, T. A.; CARNEIRO, A. C. O. Propriedades de madeiras termorretificadas de Eucalyptus grandis e $s p$. Scientia forestalis, Piracicaba, v. 40, n. 95, p. 327-336, 2012.

ASTM D143-94, Standard Test Methods for Small Clear Specimens of Timber. EUA, 2000. 31p.

ASTM D1105, Standard Test Method for Preparation of Extractive in Wood. EUA. 2007.2p.

ASTM D1106-96, Standard Test Method for AcidInsoluble Lignin in Wood. EUA. 2007. 1p.

ASSOCIAÇÃO BRASILEIRA DE NORMAS TÉCNICAS, NBR 7190: Projeto de Estruturas de madeira. Rio de janeiro 1997

BATISTA, D. C.; MUÑIZ, G. I. B. de; José Tarcísio, S.O.; Paes, J.B.; Nisgoski, S. Effect of the Brazilian thermal modification process on the chemical composition of Eucalyptus grandis juvenile wood: Part 1: Cell wall polymers and extractives contents. Maderas. Ciencia y tecnología, v. 18, n. 2, p. 273-284, 2016. DOI: http://dx.doi.org/10.4067/S0718-221X2016005000025

BOONSTRA, M. J.; ACKER, J.; KEGEL, E.; STEVENS, E. Optimization of a two-stage heat treatment process: durability aspects. Wood Science and Technology, v. 41, n. $1, \quad$ p. $31-57$ p, 2007. DOI:

http://doi.10.1007/s00226-006-0087-4

BRITO, J. O.; SILVA, F. G.; LEÃO, M. M.; ALMEIDA, G. Chemical composition changes in Eucalyptus and Pinus woods submitted to heat treatment. Bioresource Technology, v. 99, n. 18, p. 8545-8548, 2008.

CADEMARTORI, P. H. G.; SCHNEID, E,; GATTO, D. A.; BELTRAME, R.; STANGERLIN, D. M.; Modification of Static Bending Strength Properties of Eucalyptus grandis Heat-Treated. Wood, Materials Research, v. 15, n. $6, \quad$ p. 922-927, 2012. DOI: http://dx.doi.org/10.1590/S1516-14392012005000136

CADEMARTORI, P. H. G.; MISSIO, A. L.; MATTOS, B. D.; GATTO, D. A. Effect of thermal treatments on technological properties of wood from two Eucalyptus species. Anais da Academia Brasileira de Ciências, v. 87, n. 1, p. 471-481, 2015. DOI: http://dx.doi.org/10.1590/0001-3765201520130121

CARRASCO, E. V. M.; OLIVEIRA, A. L. C.; MANTILLA, J. N. R. Influência da temperatura na resistência e no módulo de elasticidade em madeira de híbridos de Eucaliptos. Ciência Florestal, v. 26, n. 2, p. 389-400, 2016. DOI: http://dx.doi.org/10.5902/1980509822740

GARCIA, R. A.; CARVALHO, A. M.; LATORRACA, J. V. F.; MATOS, J. L. M.; SANTOS, W. A.; SILVA, R. F. M.; Nondestructive evaluation of heattreated Eucalyptus grandis Hill ex Maiden wood using stress wave method. Wood Science and Technology, v. 46, p. 41-52, 2012. DOI: http://dx.doi.org/10.1007/s00226-010-0387-6

HAKKOU, M.; PE'TRISSANS,M.; ZOULALIAN, A.; GE'RARDIN, P. Investigation of wood wettability changes during heat treatment on the basis of chemical analysis. Polymer Degradation and Stability, v. 89, n. 1, p. $1-5,2005$. DOI: http:dx//doi:10.1016/j.polymdegradstab.2004.10.017

KOCAEFE, D.; PONCSAK, S.; BOLUK, Y. Effect of thermal treatment on the mechanical compossition and mechanical proeprties of Birch and aspen. Bioresources Technology, v. 3, n. 2 p. 517-537, 2008.

MODES, K. S. Efeito da retificação térmica nas propriedades físico-mecânicas e biológica das madeiras de Pinus taeda e Eucalyptus grandis. 2010. 100f. Dissertação (Mestrado em Engenharia Florestal) Universidade de Santa Maria, Santa Maria, 2010.

MOURA, L. F.; BRITO, J. O.; SILVA JÚNIOR, F. G. Effect of thermal treatment on the chemical characteristics of wood from Eucalyptus grandis W. Hill ex Maiden under different atmospheric conditions. Cerne, v. 18, n. 3, p. 449-45, 2012. DOI: http://dx.doi.org/10.1590/S010477602012000300012

POUBEL, D. S.; GARCIA, R. A.; SANTOS, W. A.; OLIVEIRA, G. L.; ABREU, H. S. Efeito da Termorretificação nas Propriedades Físicas e químicas da madeira de Pinus caribea, Cerne, Lavras, v. 19, n. 3, p. 391-398, 2013. DOI: http://dx.doi.org/10.1590/S010477602013000300005.

SILVA, M. R. Efeito do tratamento térmico nas propriedades químicas, físicas e mecânicas em elementos estruturais de Eucalytpus citriodora, e Pinus taeda. 2012. 223f. Tese (Doutorado em ciência e Engenharia de Materiais) - Universidade de São Paulo, Escola de Engenharia de São Carlos, 2012. 
SUNDQVIST, B. Colour changes and acid formation in wood during heating. 2004. 50f Doctoral Thesis, Luleå University of Technology, Sweden. 2004.

WINANDY, J.; ROWELL, R. M. Chemistry of Wood Strength. In: Rowell, R.M. (Ed.). Handbook of wood chemistry and wood composites. Florida: CRC, p.303347, 2005.

YILDIZ, S.; GEZER, E. D.; YILDIZ, U. C. Mechanical and chemical behavior of spruce wood modified by heat.
Building and Environment, v. 41, n. 1, p. 1762-1766, 2006.

ZANUNCIO, A. J. V.; NOBRE, J. R. C.; MOTTA, J. P.; TRUGILHO, P. F.; Química e colorimetria da madeira de Eucalyptus grandis W. Mill ex Maiden termorretificada Revista Árvore, Viçosa, v. 38, n .4, p. 765-770, 2014. http://dx.doi.org/10.1590/S0100-67622014000400020. 